\title{
BRECHAS TECNOLÓGICAS Y RESTRICCIÓN EXTERNA EN ARGENTINA DURANTE LOS TEMPRANOS 2000: UN ANALISIS HEURÍSTICO*
}

\author{
DOI: $10.22395 /$ seec.v21n47a5 \\ Martín Abeles* \\ Pablo Lavarello*** \\ Haroldo Montagu ${ }^{* * * *}$
}

Recibido: 05 de octubre de 2017 • Aprobado: 10 de abril de 2018

\section{RESUMEN}

El objeto de este trabajo es discutir los determinantes profundos de la restricción externa (RE) a la luz de la experiencia argentina durante la primera década de los años 2000. Se discute la hipótesis según la cual la RE resulta de la tensión existente entre una especialización basada en la eficiencia ricardiana y la eficiencia schumpeteriana. A partir de un abordaje teórico-metodológico de carácter heurístico, se busca analizar esta tensión para un periodo de alto crecimiento que coincidió con el auge en los precios internacionales de las materias primas. Como principal conclusión se arriba a que esta tensión condicionó, ante la exigüidad del cambio estructural, la posibilidad de sostener la tendencia a la reducción de las brechas sociales.

\section{PALABRAS CLAVE}

Restricción externa; eficiencia schumpeteriana; eficiencia ricardiana; cambio tecnológico; cambio estructural; desarrollo.

\section{CLASIFICACIÓN JEL}

$$
011-013-014
$$

\section{CONTENIDO}

Introducción; 1. Marco teórico: de la eficiencia ricardiana a la schumpeteriana; 2. Los determinantes de la RE: brecha tecnológica y perfil de especialización; 3 . Heterogeneidad estructural y dispersión salarial: la industria manufacturera en su encrucijada; 4. Conclusiones; Bibliografía.

\footnotetext{
Este trabajo se basa parcialmente en desarrollos preliminares del documento de los autores "Heterogeneidad estructural y restricción externa en la economía argentina" en Infante, Ricardo y Pascual Gerstenfeld (Eds.) (2013), Hacia un desarrollo inclusivo. El caso de la Argentina (LC/L.3569). Santiago de Chile: Cepal, Organización, Internacional del Trabajo (OIT).

*- Licenciado en economía, Universidad de Buenos Aires (UBA), Buenos Aires, Argentina. PhD. en Economía, New School University, Nueva York, EE. UU. Director de la oficina de la Cepal en Buenos Aires, Argentina; y Director de la Maestría en Desarrollo Económico del Idaes-Unsam, Buenos Aires, Argentina. Editor Review of Keynesian Economics (ROKE). Dirección: Paraguay 1178, 2do piso, C1057AAR, Ciudad Autónoma de Buenos Aires, Argentina. Teléfono: (54) 115436 4000. martin.abeles@cepal.org

*** Licenciado en Economia, Universidad Nacional de La Plata, Argentina. Ph. D., Université Paris XIII, Francia. Investigador CEUR-Conicet y coordinador del área de Economía Industrial, MDE-Unsam. Dirección: calle 13 n. ${ }^{\circ}$ 109, La Plata (1900), Argentina. Teléfono : 25421 4234264. Correo electrónico: plavarello@conicet.gov.ar

*** Licenciado en economía, Universidad de Buenos Aires, Buenos Aires, Argentina. MA in Economics of Development, Institute of Social Studies (Erasmus University of Rotterdam), La Haya, Holanda. Teaching Assistant, Institute of Social Studies (Erasmus University of Rotterdam), La Haya. Dirección: Kortenaerkade 12, CP: 2518AX, La Haya, Holanda. Correo electrónico: 66398HMO@eur.nl
} 


\title{
TECHNOLOGICAL GAPS AND EXTERNAL RESTRICTION IN ARGENTINA DURING THE EARLY 2000s: A HEURISTIC ANALYSIS
}

\begin{abstract}
The purpose of this paper is to discuss the deep determinants of the external restriction in the light of the Argentine experience during the first decade of the 2000s. The hypothesis according to which ER results from the tension between a specialization based on Ricardian and Schumpeterian efficiency is discussed .Based on a theoretical-methodological approach that is heuristic in nature, the aim is to analyze this tension in a high growth period that coincided with the boom in the international prices of raw materials. The main conclusion is that, given the lack of structural change, this tension conditioned the possibility of sustaining the tendency to reduce social gaps.
\end{abstract}

\section{KEYWORDS}

External restriction; Schumpeterian efficiency; Ricardian efficiency; technological change; structural change; developing.

\section{JEL CLASSIFICATION}

$011-013-014$

\section{BRECHAS TECNOLÓGICAS E RESTRIÇÃO EXTERNA NA ARGENTINA DURANTE O ANO 2000: UMA ANÁLISE HEURÍSTICA}

\section{RESUMO}

O objetivo deste artigo é discutir os determinantes profundos da restrição externa (RE) a partir da experiência argentina durante a primeira década dos anos 2000. Discute-se a hipótese segundo a qual a RE resulta da tensão existente entre uma especialização com base na eficiência ricardiana e outra com base na eficiência schumpeteriana. A partir de uma abordagem teórico-metodológica de natureza heurística, busca-se analisar essa tensão por um período de alto crescimento que coincidiu com o auge dos preços internacionais das matérias-primas. A principal conclusão é que essa tensão condicionou, dada a falta de mudança estrutural, a possibilidade de sustentar a tendência de redução das brechas sociais.

\section{PALAVRAS-CHAVE}

Restrição externa; eficiência schumpeteriana; eficiência ricardiana; mudança tecnológica; mudança estrutural; desenvolvimento.

\section{CLASSIFICAÇÃO JEL}

$$
011,013,014 .
$$

\section{CONTEÚDO}

Introdução; 1. Marco teórico: da eficiência ricardiana à eficiência schumpeteriana; 2. Determinantes da RE: brecha tecnológica e perfil de especialização; 3. Heterogeneidade estrutural e dispersão salarial: a indústria manufatureira em um dilema; 4. Conclusões; Bibliografia. 


\section{INTRODUCCIÓN}

El objeto de este trabajo es discutir los determinantes profundos de la restricción externa (RE) a la luz de la experiencia de la Argentina durante la primera década de los años 2000. Estos determinantes tienen su origen en la tensión existente entre una especialización basada en la eficiencia ricardiana asociada a las ventajas comparativas naturales y la eficiencia schumpeteriana, explicada por el potencial de aumento de productividad y de rendimientos crecientes. En los países centrales, esta tensión se resuelve en forma gradual; mientras que en los países periféricos, las diferencias persistentes de productividad entre los sectores con ventajas ricardianas y los sectores con potencial de rendimientos crecientes son de tal magnitud que es difícil avanzar en procesos de cambio estructural sin afectar los incentivos de mercado, y sin procesos acelerados de aprendizaje tecnológico en las nuevas actividades.

Como lo evidenció el comportamiento de la economía argentina en el periodo 2003-2015, es difícil que la dificultad de resolver esta tensión que se encuentra en el origen de la RE como limitante del crecimiento desaparezca sin cambios mayores en la estructura productiva. Los pioneros de la literatura estructuralista del desarrollo, incluidos los iniciadores del pensamiento de la Cepal, discutieron este tipo de condicionantes en el contexto histórico de la segunda postguerra. Por ejemplo, de acuerdo con el aporte seminal de Prebisch (1949), el crecimiento de los países de la periferia se encontraba limitado por la modalidad de inserción en la división internacional del trabajo bajo dos modalidades: la tendencia a la caída de los términos de intercambio de los países especializados en la exportación de materias primas, mecanismo que resultaba en una distribución desigual de los frutos del progreso técnico entre los países centrales y periféricos; y el carácter heterogéneo de la estructura productiva, derivado de la difusión asimétrica del progreso técnico que se veía focalizado en actividades en las que se contaba con ventajas comparativas.

Como consecuencia de esta modalidad de inserción en la economía internacional, el desarrollo del centro evidenciaba un mayor grado de homogeneidad en sus productividades sectoriales y una relativamente mayor diversificación de su aparato productivo; mientras que la periferia tendía a evidenciar un alto grado de especialización en las actividades que adoptaban las tecnologías del centro y una elevada heterogeneidad de sus productividades, que se profundizaban con el tiempo. En línea con este enfoque, Pinto (1970) desarrolló el concepto de heterogeneidad estructural, que contribuyó a caracterizar las deficiencias y debilidades del proceso de industrialización sustitutiva de posguerra. Como lo apunta Sunkel (1970), dicha heterogeneidad (con especificidades propias a cada periodo histórico) se retroalimentan de manera dinámica por la interacción entre las estructuras del centro y la 
periferia mediante diversos mecanismos acumulativos en lo tecnológico, lo económico, lo social y lo político.

Estos conjuntos de aportes marcarían un importante avance en la comprensión de los factores estructurales que condicionan los procesos de desarrollo, parte de los cuales se expresan en la persistencia de la restricción externa. Desde la década de 1980, la literatura estructuralista tiende a confluir con las contribuciones de la literatura neoschumpeteriana-evolucionista, en tanto que avanza en la comprensión de los procesos de aprendizaje tecnológico y organizacional que explican la microdinámica de estos comportamientos agregados y la reproducción de la heterogeneidad estructural (Katz, 1967, 1978, 1984; Chudnovsky, 1985; Chudnovsky y Lopez, 2007; Cimoli y Porcile, 2011).

Las revoluciones tecnológicas que tomaron impulso a partir de la década de 1970, con el nuevo paradigma de la microelectrónica (y más tarde con las tecnologías de la información y la biotecnología), implicaron desafíos mayores para los países de la periferia, en la medida que la difusión asimétrica del progreso técnico trajo nuevas tensiones entre el perfil de especialización existente y las oportunidades de las nuevas tecnologías. Desde esta perspectiva, las brechas tecnológicas asumen una importancia creciente en la participación de cada país en los flujos internacionales de comercio e inversión, y a través del efecto de estos flujos en la competitividad estructural, en la máxima tasa de crecimiento compatible con la restricción externa que cada país puede alcanzar. La importancia de las asimetrías tecnológicas entre países revela la tensión de las ventajas absolutas asociadas a la eficiencia schumpeteriana vis a vis las ventajas comparativas estáticas (Dosi, Tyson y Zysman, 1989). Estas últimas no serían más que la consecuencia del nivel de ventajas absolutas que cada país obtiene en aquellos sectores que, por la presencia de rendimientos crecientes dinámicos, definen la competitividad de la economía en el comercio internacional (Dosi, 1988).

Pese a lo anterior, los círculos virtuosos entre la tecnología, la competitividad y las tasas de crecimiento no son totalmente automáticos y endógenos al proceso de desarrollo. En este sentido, la literatura estructuralista plantea que la reducción de las brechas tecnológicas en un conjunto de actividades modernas orientadas a la exportación de materias primas puede reforzar las diferencias intersectoriales de productividades a través de efectos de encerramiento (lock in), con el consecuente bloqueo de la diversificación de las exportaciones y de la estructura productiva, y la profundización de la dependencia de las importaciones manufactureras.

Tal persistencia de la especialización y la heterogeneidad estructural presenta importantes dilemas que trascienden lo estrictamente económico: ponen en el 
centro del debate la dificultad para consolidar un sendero de desarrollo que concilie el crecimiento con la disminución de las asimetrías salariales, de modo tal que se plantee una verdadera encrucijada para un país en el que la expansión de derechos de los trabajadores se ha transformado en el móvil prioritario de la legitimidad de la democracia.

En este artículo se buscará ilustrar, en clave heurística, la presencia de esta tensión entre la eficiencia ricardiana y la eficiencia schumpeteriana en Argentina durante el periodo 2003-2008 -el de mayor crecimiento de las últimas décadas-, que coincidió con el auge en los precios internacionales de las materias primas; y cómo esa tensión, ante la exigüidad del cambio estructural, a la postre condicionó la posibilidad de sostener la tendencia a la reducción de las brechas sociales.

\section{MARCO TEÓRICO: DE LA EFICIENCIA RICARDIANA A LA SCHUMPETERIANA}

El abordaje dinámico del problema de la RE parte de la distinción entre la eficiencia ricardiana o asignativa (allocative efficiency), asociada al concepto ricardiano de ventajas comparativas, de otros tipos de eficiencia, como la keynesiana (o de crecimiento) y la schumpeteriana (o tecnológica), tal cual fue planteada por la literatura neoschumpeteriana-evolucionista (Dosi, Tyson y Zysman, 1989).

De acuerdo con un patrón de especialización basado en ventajas comparativas, que asigna la inversión entre las distintas actividades al interior de un espacio nacional en función de sus productividades relativas, cada país tenderá a especializarse en aquellas ramas en las que presenta menores brechas de productividad respecto del resto del mundo. Desde una perspectiva schumpeteriana, partiendo del reconocimiento de diferencias entre distintas ramas en el potencial de rendimientos crecientes (internos y externos), no es posible asegurar que la especialización ricardiana basada en las ventajas comparativas (estáticas) resulte compatible con una especialización fundada en los otros dos tipos de eficiencia.

Desde una perspectiva dinámica, ya sea keynesiana o schumpeteriana, es necesario tener en cuenta los efectos del patrón de especialización sobre las tasas de crecimiento compatibles con la restricción externa (eficiencia keynesiana), así como en función de su consistencia con aquellas direcciones del cambio tecnológico con mayor potencial de rendimientos crecientes. Esta última da lugar a la denominada eficiencia schumpeteriana, basada en las ventajas absolutas asociadas a las oportunidades de aprendizajes tecnológicos en los distintos sectores.

Desde un legado keynesiano, los desarrollos analíticos más relevantes se basaron en la existencia de una regularidad empírica que expresa la articulación de los deter- 
minantes del crecimiento originados en el lado de la demanda con la noción de RE, tal como lo desarrolló en su artículo pionero Anthony Thirlwall (1979). Según esta regularidad empírica, que se conoce como ley de Thirlwall, la máxima tasa de crecimiento posible de una economía en el largo plazo, dado un ritmo de crecimiento de la demanda mundial, se encuentra directamente relacionada con la elasticidad de las exportaciones respecto del crecimiento de dicha demanda; e inversamente relacionada con la elasticidad-ingreso de las importaciones respecto del ingreso del país considerado. En su versión inicial, que supone dados los términos de intercambio (de esta manera excluyendo las elasticidades de las exportaciones y las importaciones respecto a los precios internacionales), la ley de Thirlwall puede expresarse en la siguiente ecuación:

$$
\mathrm{y}^{*}=\varepsilon z / \pi
$$

donde $y^{*}$ es la tasa de crecimiento sostenible de la economía en el largo plazo; $\varepsilon$, la elasticidad-ingreso mundial de las exportaciones; $z$, la tasa de crecimiento del resto del mundo; y $\pi$, la elasticidad-ingreso de las importaciones.

Como se discute en Abeles et al. (2013), la ley de Thirlwall postula una restricción o equilibrio de largo plazo. Es decir, deja abierta la posibilidad de que el ritmo de crecimiento de equilibrio establecido por los parámetros $\varepsilon$, z y $\pi$ no sea alcanzado (o sea sobrepasado) a corto o mediano plazo, hecho que dará lugar a la acumulación de reservas internacionales (o al endeudamiento mediante el acceso a financiamiento externo). Sin embargo, pone de manifiesto que un periodo de crecimiento relativamente acelerado (vis a vis el umbral establecido por dichos parámetros) debe, eventualmente, ser compensado por un periodo de crecimiento relativamente lento (vis a vis el mismo umbral); esto último a menos que, en el ínterin, la RE se haya desplazado "hacia afuera" debido a la modificación de las características estructurales de la economía ( $\varepsilon$ y $\pi$ ) o al aumento en el ritmo de crecimiento de la economía mundial (z). Desde la perspectiva de este trabajo, es relevante vincular el problema de la RE expresada en la ley de Thirlwall con la estructura económica a través de la especificación de los determinantes de las elasticidades-ingreso de importaciones y exportaciones, dos parámetros medulares de todo aparato productivo (Cimoli, Porcile y Rovira, 2009) .

Un estudio reciente de la Cepal ha postulado en forma sintética que la tasa de crecimiento consistente con la RE depende de las características de la estructura productiva y de la brecha tecnológica con las economías industrializadas (brecha tecnológica externa) (Cepal, 2007). Este trabajo parte de la ley de Thirlwall y plantea, a modo de hipótesis, que las elasticidades de exportaciones e importacio- 
nes $(\varepsilon$ y $\pi)$ dependen de tres factores estructurales o institucionales: el grado de especialización y heterogeneidad de la estructura productiva (E); la brecha tecnológica externa $(\psi)$; y otros factores ajenos a estructura económica y a la tecnología, relacionados de modo fundamental con la determinación de los precios relativos de la economía $(\Omega)$. A partir de esta línea interpretativa, en su forma implícita la ley de Thirlwall puede reexpresarse de la siguiente manera:

$$
y^{*}=\varepsilon / \pi(E, \psi, \Omega) z
$$

De esta manera, la RE se encuentra determinada por parámetros más profundos, vinculados con el carácter heterogéneo y especializado de la estructura productiva, en línea con la tesis de Raúl Prebisch (1949; 1962). Por su parte, la persistencia de la heterogeneidad estructural de las economías en desarrollo señalada por Pinto (1970) tiende a reproducir un patrón de especialización concentrado en un conjunto limitado de commodities, las cuales cuentan con ventajas comparativas, o en actividades de carácter industrial protegidas. En estas últimas, frente las asimetrías de capacidades vis a vis los países desarrollados tienden a importar insumos de mayor elasticidad ingreso a medida que aumenta el ritmo de crecimiento, proceso que la estrategia de sustitución de importaciones, tal como fue adoptada en los países de América Latina, exacerbó en lugar de resolver.

Desde una perspectiva schumpeteriana, las ventajas comparativas solo son una expresión ex post de las ventajas absolutas de las industrias en el marco de la competencia internacional. A ello se suma la constatación de que el principal determinante de las ventajas absolutas es la innovación, resultado de las actividades de investigación y desarrollo (IyD) y de los procesos de aprendizaje en el proceso productivo (Dosi y Soete, 1988). Estos aprendizajes incluyen tanto el aprendizaje por la experiencia en la producción (learning by doing) como aquel que resulta de la interacción entre proveedores y usuarios. Una consecuencia directa de este abordaje radica en que distintas especializaciones productivas en el presente se traducen en oportunidades diversas de aprendizaje tecnológico y, derivado de lo anterior, en diferentes capacidades tecnológicas y productividades en los periodos futuros. Como las oportunidades tecnológicas varían de manera intersectorial, el patrón de especialización productiva en el presente puede tener efectos decisivos en las productividades absolutas de los distintos sectores y, por ende, en las productividades relativas en el futuro.

Para los países en desarrollo, esto plantea posibilidades de tensiones entre la asignación de recursos según la eficiencia ricardiana y la que resulta de una especialización schumpeteriana. Esto tiene consecuencias en materia de política indus- 
trial. Aceptar los precios relativos que establece el mercado internacional tiende a reforzar la rentabilidad de los sectores que presentan menores brechas de productividad con respecto a los países desarrollados. Si bien esta estructura de rentabilidades es más eficiente desde una perspectiva de ventajas comparativas estáticas, la misma puede limitar el potencial de aumentos de productividad a mediano y largo plazos. Por un lado, como se trata de los sectores con menor brecha de productividades respecto a las mejores prácticas internacionales, resulta menor el potencial de aumento de productividad derivado de los procesos de catching up (convergencia). A su vez, como dichos sectores no necesariamente corresponden a las tecnologías con mayor potencial de progreso técnico (y aumento de la productividad) a escala internacional (situación típica de los sectores intensivos en recursos naturales [RR. NN.] que predominan en la región), el perfil de especialización resultante puede limitar el crecimiento no solo por el bajo potencial de convergencia, sino también por su mínima proyección de mediano o largo plazos. Una especialización en actividades "maduras" de mayor productividad relativa, es decir, con una mayor ventaja comparativa, un bajo ritmo de progreso técnico y un reducido margen de convergencia, puede derivar en ritmos de crecimiento insatisfactorios de la productividad total.

A fin de ilustrar este punto, ciertos autores han partido del modelo original de Dornbusch, Fischer y Samuelson (1977) y han introducido las contribuciones schumpeterianas y cepalinas (Cimoli, 1988; Cimoli y Porcile, 2011), para lo cual consideran una economía internacional formada por dos países - del centro y la periferia- en la que se produce un gran número de bienes $\mathrm{N}$, y se supone que cada uno de ellos requiere para su producción una cantidad de horas de trabajo igual a en la periferia y en el centro.

El cociente entre los requerimientos de trabajo para producir el bien i en el centro y la periferia $\mathrm{A}$ = representa la productividad relativa de la periferia respecto al centro requerida para producir el bien $i$, tal que cuanto mayor sea A, menor será la brecha de productividad para el bien $i$; y ceteris paribus, mayor será la ventaja comparativa. En el primer cuadrante del gráfico, 1, los $\mathrm{N}$ bienes se ordenan en una secuencia decreciente de acuerdo con la productividad relativa de la periferia de tal modo que, para $\mathrm{N}=1$, la periferia tiene la mayor productividad relativa; y a medida que se diversifica la economía hacia los productos subsiguientes, la productividad relativa es menor. A medida que se avanza sobre la recta A hacia productos más intensivos en conocimiento, para una brecha tecnológica dada, la brecha de productividad es mayor y esto se refleja en las productividades relativas. La altura de la recta A depende de la brecha tecnológica respecto del centro, es decir, de las diferencias en las capacidades para asimilar y generar nuevas tecnologías. 


\section{Gráfico 1. Brecha tecnológica y ley de Thirlwall}

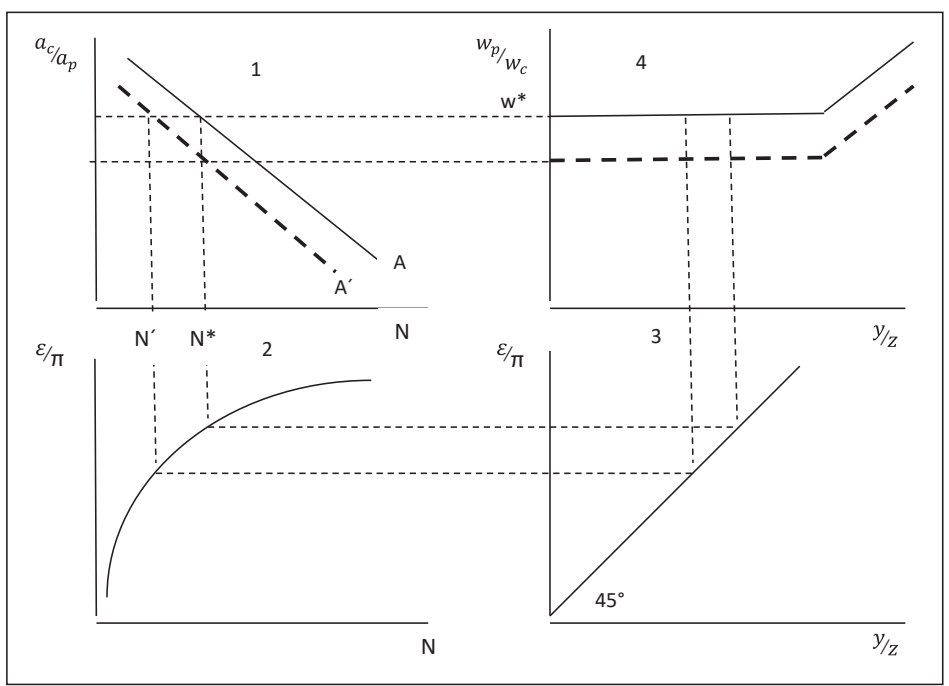

Fuente: elaboración propia a partir de Cimoli y Porcile (2011).

Al introducir los salarios relativos de la periferia respecto al centro, es posible definir el perfil de especialización de la periferia. En este modelo, el precio de los bienes se define a partir de su costo unitario, dado por el salario y el requerimiento de trabajo'. En consecuencia, la periferia producirá hasta aquel bien $\mathrm{N}$ para el cual , lo cual se puede reescribir (como en el gráfico 1). La periferia producirá hasta aquel bien en el que la productividad relativa de la periferia sea mayor o igual que su salario relativo. Para un nivel de $\mathrm{w}^{*}$ en la figura 1, la periferia producirá entre el bien $\mathrm{N}=1$ y $\mathrm{N}^{*}$; mientras que el centro producirá a partir de $\mathrm{N}^{*}$, indicando el grado de diversificación de la periferia. Como el centro produce los bienes mayores que $\mathrm{N}^{*}$ de mayor intensidad tecnológica (y como lo veremos en los siguientes párrafos, de mayor dinamismo de la demanda), el perfil de especialización de la periferia operará como restricción sobre el crecimiento.

Para especificar cómo operan estas restricciones, en el cuadrante 2 se representa la relación entre el patrón de especialización productiva de la periferia (esto es, el valor de $\mathrm{N}^{*}$ ) y el cociente entre la elasticidad-ingreso de la demanda de exportaciones (del país periférico) y aquella de la demanda de importaciones, es decir, $\varepsilon / \pi^{2}$. Se supone que la relación entre las elasticidades depende de forma positiva

\footnotetext{
1 Sería posible incorporar los márgenes de ganancia a partir de un mark up, sin modificar las conclusiones del modelo.

2 En el marco de la actual representación de una economía periférica y una central, $\pi$ es la elasticidad-ingreso mundial de las exportaciones del centro.
} 
de la diversificación productiva hacia sectores de mayor complejidad tecnológica de la periferia. La idea subyacente detrás de esta relación funcional consiste en que, si existe un patrón de especialización en el que los sectores con mayor intensidad tecnológica tienen mayor peso (cuestión que se ilustra con un desplazamiento a mayores N), esto permite al país contar con capacidades tecnoproductivas para imitar las últimas innovaciones del centro y entrar en mercados de productos que, dada su fase inicial del ciclo de producto, manifiestan un alto crecimiento de la demanda interna y externa. De esta manera queda especificada la relación entre el perfil de especialización y la dinámica de la demanda, tal como se representa en el segundo cuadrante del gráfico 1 .

$$
\varepsilon / \pi=f\left(N^{*}\right), \operatorname{con}^{\prime}>0
$$

La ley de Thirlwall, entendida como una condición de equilibrio en el largo plazo, se presenta en el tercer cuadrante. La tasa relativa de crecimiento de la periferia respecto al centro será $y / z$, en donde $y$ es la tasa de crecimiento de la periferia y $z$ la tasa de crecimiento del centro debe ser igual al cociente de elasticidades $\varepsilon / \pi$. La recta de $45^{\circ}$ ilustra esta condición de equilibrio, en tanto sobre la misma los cocientes de elasticidades consistentes con distintos patrones de especialización son iguales al cociente de tasas de crecimiento de equilibrio de ambas economías.

Por último, se introducen las especificidades del mercado de trabajo en la periferia. A tal fin se postula que la relación entre la tasa de crecimiento relativa de la periferia y los salarios relativos difiere de la existente en un país central. Debido a ello, en el cuadrante 4 se establece que, en los países de la periferia donde existe heterogeneidad en el mercado de trabajo con una proporción elevada de la fuerza de trabajo desempleada o empleada informalmente, el salario relativo no aumenta ante el mayor crecimiento relativo. Solo a partir de cierta tasa de crecimiento relativa de la periferia, el salario relativo centro-periferia responde de forma positiva al crecimiento relativo de la periferia.

De esta manera se determina el crecimiento relativo de equilibrio de un país periférico para un nivel de brecha tecnológica, heterogeneidad productiva y empleo. A partir de esta situación es posible interrogarse cómo una ampliación de la brecha tecnológica para distintos grados de heterogeneidad estructural afecta las tasas de crecimiento consistentes con el equilibrio externo.

Cimoli y Porcile (2011) plantean esta posibilidad de forma simplificada. Una ampliación de la brecha tecnológica resulta en un desplazamiento de la curva A hasta A $^{\prime}$ en el cuadrante 1 del gráfico 2. Para el nivel de salarios relativos $\mathrm{w}^{*}$, esto implica una mayor especialización de la estructura productiva en sectores con menor di- 
namismo tecnológico (de $\mathrm{N}^{*}$ a $\mathrm{N}^{\prime}$ ) y menores elasticidades relativas de la demanda, por lo que las tasas de crecimiento consistentes con la restricción externa resultarán menores. Una devaluación que reduzca de forma relativa los salarios de la periferia podría compensar este efecto, desplazándose la recta de salarios $\mathrm{w}^{*}$ del cuarto cuadrante hacia abajo. Como lo afirman Cimoli y Porcile (2011), esto lograría mantener el grado de diversificación previo, pero solo como resultado de una mejora "espuria" de la competitividad que implicaría una caída del salario real de la periferia (o con efectos transitorios e inflacionarios en la medida en que los trabajadores logren recomponer su poder adquisitivo).

Este modelo simplificado permite identificar el efecto de una mayor heterogeneidad estructural en el perfil de especialización. Como se puede apreciar en el gráfico 2, cuanto más heterogénea es la estructura productiva, más inclinada es la recta A, porque al pasar de un grado de diversificación $\mathrm{N}$ a otro $\mathrm{N}+1$, la productividad relativa es significativamente menor. En ese contexto, la disminución del salario relativo asociada a una devaluación de la moneda de la periferia tiene un efecto menor sobre la diversificación productiva (de $\mathrm{N}^{*}$ a $\mathrm{N}^{\prime \prime}$ en vez de alcanzar $\mathrm{N}^{\prime}$ ), y ceteris paribus, sobre la tasa de crecimiento consistente con la restricción externa. Esta situación refleja la modalidad de inserción de las economías periféricas en la economía mundial. De esta manera se puede ver cómo la difusión del progreso técnico en estos países se manifiesta de manera incompleta y asimétrica; se focaliza en los sectores en los que ya se cuenta con ventajas comparativas, con lo cual aumenta la heterogeneidad estructural.

\section{Gráfico 2. Brecha tecnológica y heterogeneidad estructural}

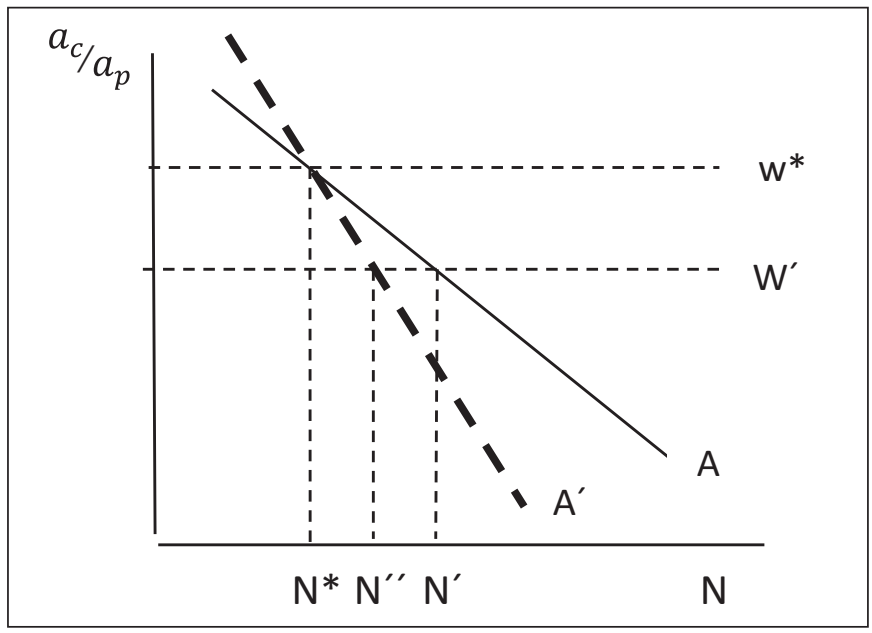

Fuente: elaboración propia a partir de Cimoli y Porcile (2011). 
Siguiendo el análisis de Abeles et al. (2013) es posible plantear como corolario que una política de apoyo a la adopción de tecnologías de carácter horizontal favorecerá solo a aquellos sectores en los que ya se cuente con una menor brecha tecnológica relativa, con lo que se profundizará la heterogeneidad estructural.

De esta manera se desarrollan, a partir de un esquema simplificado, las relaciones que se encuentran detrás de la expresión reducida (2): las elasticidades de importación y exportación (el cociente entre $\varepsilon$ y $\pi$ ) dependen, en última instancia, de las brechas tecnológicas intersectoriales entre el país considerado y los países avanzados; del grado de heterogeneidad estructural de la economía; y de un conjunto de variables que, de una u otra manera, inciden en la competitividad de la economía, aunque estas últimas variables no estén relacionadas de modo directo con factores tecnológicos (desde la política monetaria, cambiaria y de financiamiento hasta la estructura arancelaria y de subsidios correspondiente a los distintos sectores productivos) $)^{3}$.

\section{LOS DETERMINANTES DE LA RE: BRECHA TECNOLÓGICA Y PERFIL DE ESPECIALIZACIÓN}

En la sección anterior se presentaron los determinantes de la restricción externa desde un punto de vista analítico, teniéndose en cuenta los desarrollos teóricos más relevantes referidos al tema en cuestión. Ahora bien, ante la pregunta de si la RE en Argentina y la región se ha relajado o profundizado, es necesario, dentro del marco conceptual expuesto, analizar la evolución de las brechas tecnológicas sectoriales. El carácter persistente de la RE como limitante del crecimiento en la economía argentina tiene sus determinantes en la persistencia de un perfil de especialización basado en las ventajas comparativas. En esta sección se ilustrará de forma empírica cómo, más allá de ciertos cambios acotados en la estructura productiva, la reducción de la brecha tecnológica se limitó a ciertos sectores, hecho que dio lugar a una mayor especialización y heterogeneidad de dicha estructura. De esta manera, y mediante un abordaje de teoría apreciativa, se busca comprender la evolución de la estructura productiva y las brechas tecnológicas; se parte para ello de la hipótesis de Prebisch respecto al carácter desigual e incompleto de la difusión del progreso técnico como mecanismo de reproducción de la condición periférica, a partir de la experiencia reciente de Argentina.

A pesar de las dos experiencias de liberalización financiera -apertura cum apreciación cambiaria entre mediados de la década de 1970 e inicios de los años 2000-,

López y Cruz (2000) muestran para el caso de los países latinoamericanos cómo una política de sostenimiento del tipo de cambio en un nivel competitivo puede elevar el ritmo de crecimiento compatible con la RE. 
la estructura económica argentina mantuvo durante todo el periodo una participación de la industria manufacturera superior a la del conjunto de países de América Latina (LAC 10). La comparación de los cambios en la estructura productiva muestra que estos rasgos específicos de la economía argentina no llegaron a revertirse, a pesar de condiciones internacionales que favorecieron una especialización hacia los sectores primarios y no transables. Como se puede apreciar en el gráfico 3 (a), los países de América Latina evidencian una tendencia al aumento de la participación de los sectores primarios en la estructura económica argentina desde inicios de la década de 1990: de modo concreto, el sector primario aumentó su participación de 13,3 \% a 15,0 \% entre 1990 y 2003, para luego descender un punto porcentual y medio hacia fines de la década del 2000. Si bien Argentina no fue ajeno al mencionado proceso de reprimarización de su estructura productiva durante dicha década, entre el año 2003 y 2008 esta tendencia se detuvo con mayor fuerza que en el conjunto de los países de América Latina; pasó de 8,4 \% a 6,2 \% del PIB.

El sector servicios, que pierde peso en la estructura económica del LAT-10, tendió a aumentar su participación en el valor agregado durante la década de 1990 en Argentina. Esta tendencia también se detuvo en el periodo analizado, al pasar de $65,1 \%$ a 61,1 \% entre 2002 y 2008. Como contrapartida de esta dinámica de menor peso de las actividades primarias y de servicios, en ese periodo se vio frenado de forma leve el proceso de desindustrialización, aunque sin recuperar el peso de la industria manufacturera existente en el inicio de la década de 1990. Si bien resulta incipiente y limitado, este comportamiento debe evaluarse en el marco de una tendencia regional a la desindustrialización.

Estas evoluciones muestran que la industria manufacturera argentina ha logrado contener la desindustrialización que caracterizó a la región. No obstante, el análisis de estos comportamientos agregados no permite dar cuenta de los cambios que se experimentaron al interior de los sistemas manufactureros, que en la actualidad se estructuran en redes globales de producción. Como resultado de estos cambios, al interior de la industria coexisten, por un lado, actividades tradicionales de ensamble con alto grado de dependencia de proveedores de equipos e insumos; y por otro, actividades de componentes intensivas en ingeniería con mayores oportunidades de aprendizajes y efectos de difusión intersectorial del progreso técnico. Esto exige distinguir entre distintos tipos de industrias al interior de la industria manufacturera, a fin de tener en cuenta el cambio estructural.

En aras de avanzar en un análisis más desagregado, que permita pasar de un nivel macroeconómico a uno de subsector, o incluso de actividad, es posible apoyarse en el trabajo de Katz y Stumpo (2001): en un esfuerzo por elaborar una taxo- 
Martín Abeles - Pablo Lavarello - Haroldo Montagu

Gráfico 3. Cambios en la estructura productiva - Argentina y LAC-10

(a) Participación del VA sector primario (en porcentaje del PIB)

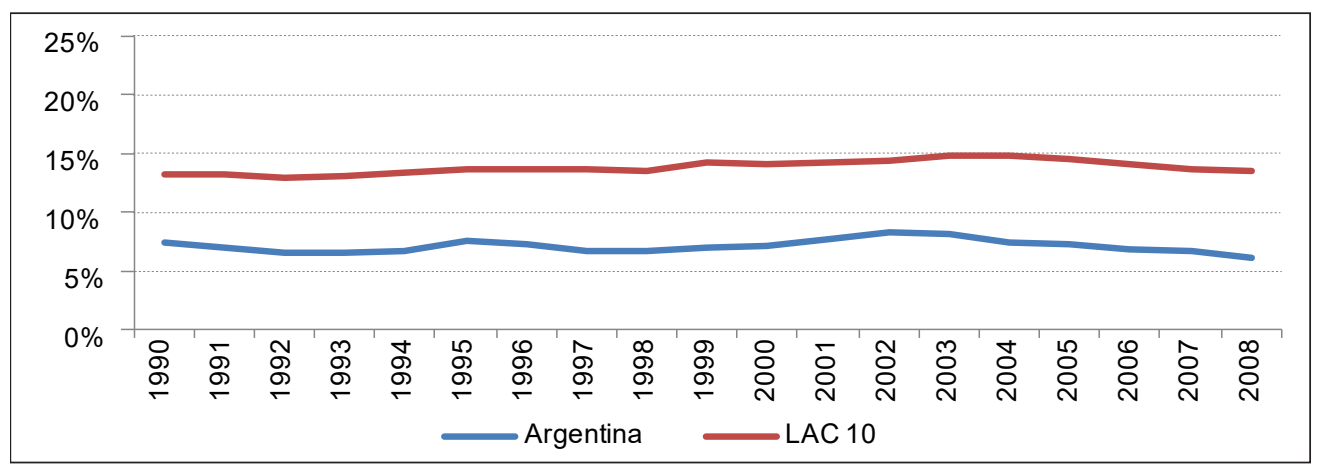

(b) Participación del VA industria manufacturera (en porcentaje del PIB)

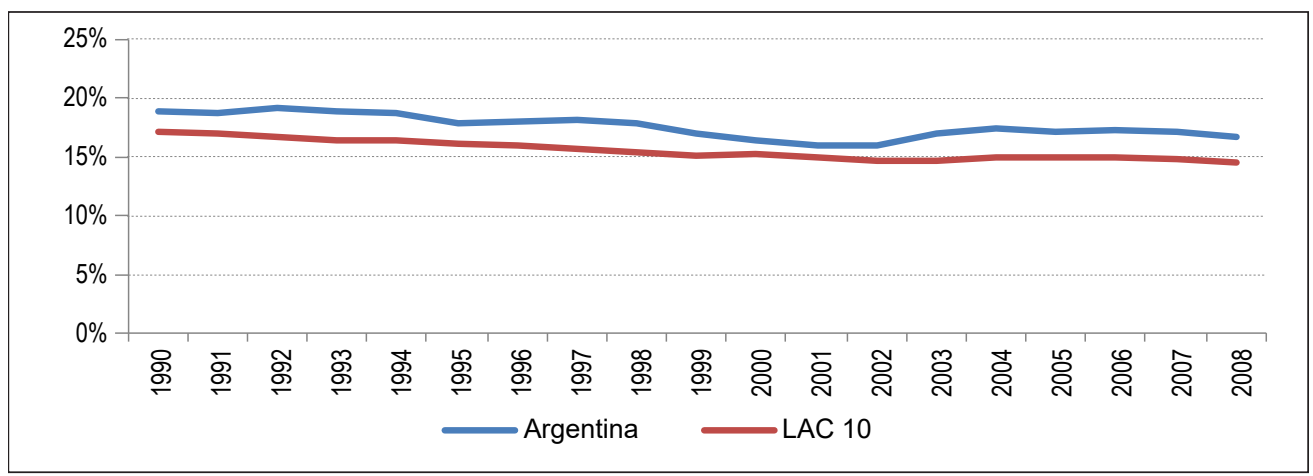

(c) Participación del VA sector de servicios (en porcentaje del PIB)

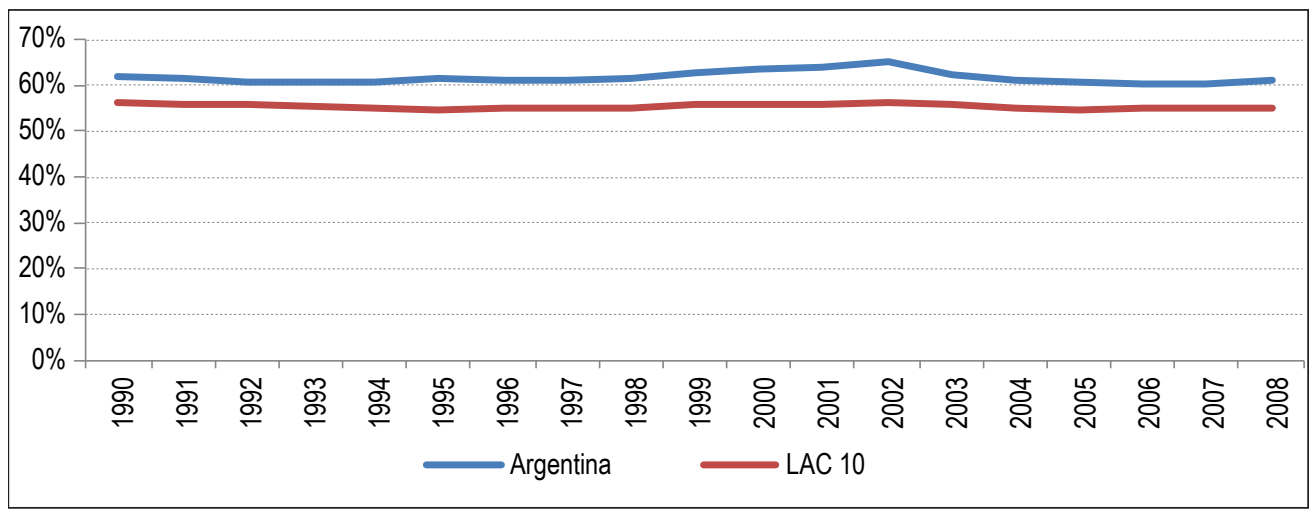

Fuente: Cepal. 
nomía de actividades adaptada a las condiciones de los países semiindustrializados, estos autores establecen una distinción entre sectores intensivos en RR. NN., en empleo y en ingeniería. Dentro de estos últimos se distinguen en este trabajo el sector automotriz del intensivo en ingeniería propiamente dicho, en tanto que este último integra las industrias metalmecánicas, y en particular, la de bienes de capital, que juega un rol clave en los procesos de aprendizaje y difusión intersectorial del conocimiento tecnológico, tal como lo ha resaltado la literatura neoschumpeteriana (Rosenberg, 1974; Patel y Pavitt, 1994).

Como se desprende del análisis realizado por Abeles et al. (2013), la evolución de largo plazo de la composición del valor agregado de la industria manufacturera argentina se caracteriza por un aumento de la participación de los sectores intensivos en RR. NN. entre los años 1973 y 2000. En el marco de una disminución en el peso de la industria manufacturera en su conjunto, las actividades intensivas en RR. NN. aumentaron su gravitación al interior de la industria en el periodo mencionado, mientras que los sectores intensivos en ingeniería disminuyeron su participación. Las industrias intensivas en RR. NN. incorporaron técnicas en las mejores prácticas internacionales a través de la adquisición de bienes de capital y plantas llave en mano, en forma simultánea a la expansión de la agricultura. Esto explica la reducción de la brecha tecnológica del sector intensivo en RR. NN. De esta manera se manifestó un patrón de industrialización basado en el desarrollo de capacidades productivas, con bajo grado de integración nacional de capacidades en actividades de ingeniería intensiva.

A partir de la década de 1990, con la vigencia del Mercosur y los cambios en las estructuras organizacionales de las empresas multinacionales (EMN) de estrategias multidomésticas a globales, se produjo una reducción de las brechas de productividad externas en la industria automotriz. Los acuerdos regionales de complementación productiva, así como la especialización por componente o producto de la EMN durante la década, posibilitaron un proceso de upgrading o ascenso tecnológico. Este trajo aparejadas la racionalización y relocalización de la mayor parte de las actividades que se realizaban en el espacio nacional durante la industrialización por sustitución de importaciones ISI. Dado el carácter selectivo de dicho proceso de reestructuración a escala global, el proceso de ascenso tecnológico nombrado - upgrading- debe tomarse con cautela, dadas las particularidades de los sectores de ensamble, que ven reducir su brecha externa de productividad al tiempo que aumenta la fragmentación de sus procesos productivos. Como lo sostienen Abeles et al. (2013), a diferencia de la reducción en las brechas de productividad a diferencia de la dinámica de dicho sector durante la ISI, en las últimas dos décadas la productividad no se explica por aprendizajes adaptativos sino por la racionalización 
Gráfico 4. Estructura productiva y brechas de productividad - Argentina y EE. UU.
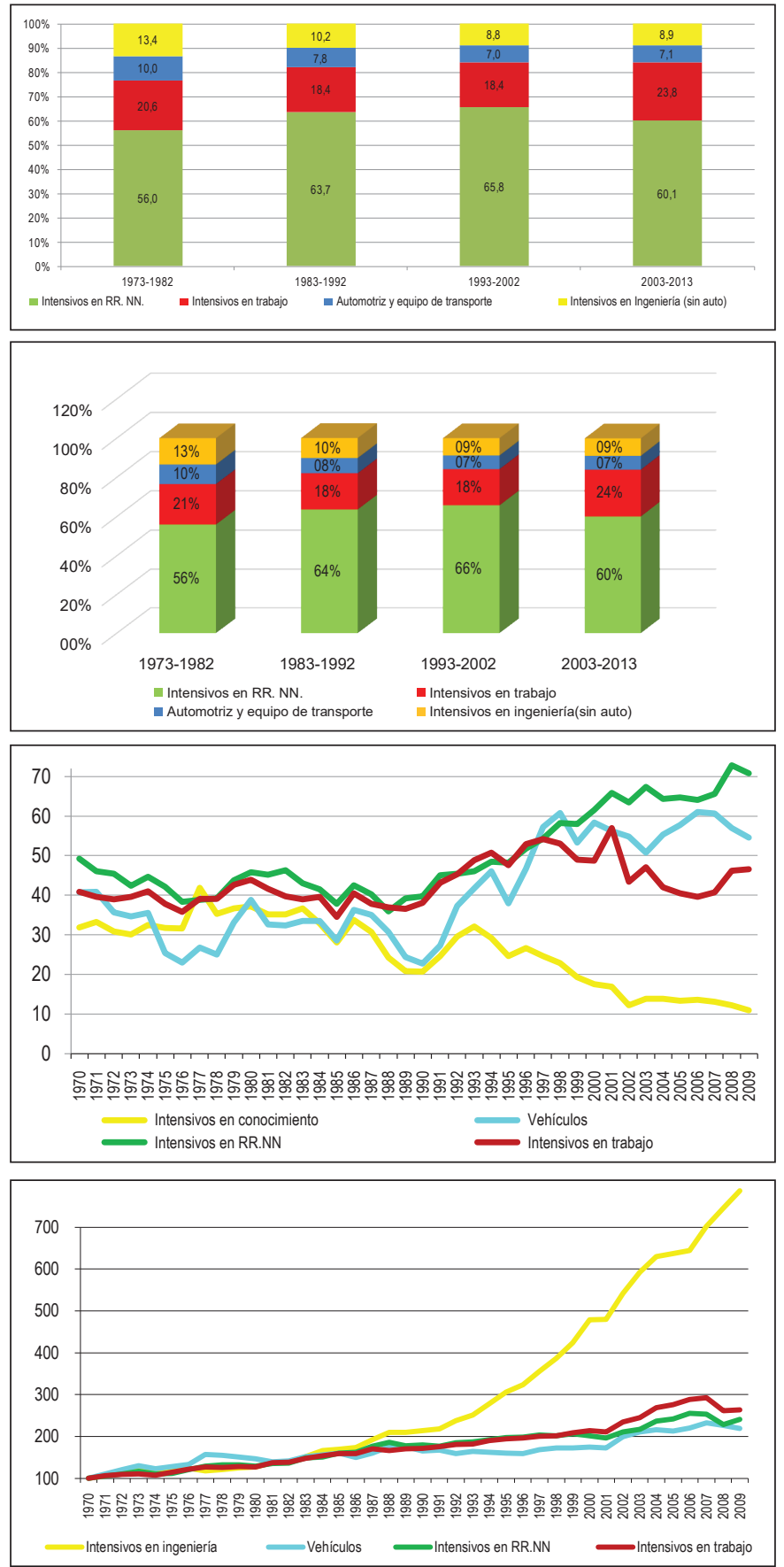

Fuente: PADI - Cepal. 
de las plantas con una deslocalización del sector autopartista hacia Brasil. No es luego el aprendizaje tecnológico previo sino los cambios en las formas de organización con una importante dosis de desintegración nacional y mayor especialización intrarregional el factor determinante de la reducción en la brecha de productividades de este sector.

A partir de la primera década de los 2000, dadas las nuevas condiciones macroeconómicas, la industria manufacturera comenzó, de manera incipiente, a revertir el largo proceso de reestructuración hacia sectores intensivos en RR. NN. ${ }^{4}$. El establecimiento de un marco de incentivos basado en tipo de cambio competitivo e impuestos a las exportaciones a los sectores intensivos en RR. NN. posibilitó un cambio en la composición del valor agregado del sector hacia actividades trabajo-intensivas y, en menor medida, de las ramas intensivas en ingeniería y conocimiento (Lavarello y Sarabia, 2017). Al mismo tiempo, los sectores intensivos en RR. NN. vieron disminuir su participación en el total de valor agregado manufacturero.

Este cambio en la estructura del valor agregado no fue acompañado por una reducción de las brechas externas de productividad (gráfico 4.b). El sector intensivo en RR. NN. mantiene su brecha en un nivel semejante al de la década de 1990. El sector automotriz también mantuvo las brechas de productividad, luego de recuperar el terreno perdido durante la contracción del periodo 1996-2001. En contraste, se evidencia una ampliación de la brecha de productividad en los sectores intensivos en trabajo e intensivos en ingeniería, punto en el que se advierte el crecimiento más importante en la participación del valor agregado. Este hecho revela uno de los principales límites del proceso de "reindustrialización" de la década del 2000.

Aun si las políticas macroeconómicas aplicadas a partir de 2003 lograron cambiar la estructura de la industria manufacturera a partir del desafío de la eficiencia ricardiana, tal como se refleja en la composición del valor agregado y del empleo, las mismas no lograron modificar las ventajas comparativas sectoriales, asociadas a la dotación de RR. NN. o a las estrategias de las EMN (Abeles y Amar, 2017). De esta forma, persisten las brechas de productividad externas y se reproducen o amplifican las internas, asociadas a la heterogeneidad estructural, y explicadas por la debilidad de los encadenamientos tecnológicos entre las industrias con ventajas comparativas y los proveedores locales de tecnología (Lavarello, 2004; Abeles et al. 2013).

Esta conclusión plantea dilemas mayores frente a la aceleración del cambio tecnológico en la actual fase de difusión de las industrias 4.0 (Cimoli et al., 2017). En cada revolución tecnológica existe un conjunto de actividades intensivas en co-

$4 \quad$ Para un análisis más exhaustivo, véanse Roitter et al. (2011) 
nocimiento que expresan mayores ritmos de progreso técnico y que, a partir de la instalación de un conjunto de diseños dominantes y nuevos modelos de negocios, generan tendencias a la homogenización intersectorial. El gráfico 4 (c) muestra que la productividad laboral de Estados Unidos creció de forma acelerada en las ramas de actividad intensivas en ingeniería a partir de la década de 1990, con la difusión de las nuevas tecnologías. En contraste, los sectores intensivos en RR. NN., trabajo y, dentro de las actividades intensivas en ingeniería, la rama automotriz, si bien aumentaron su productividad, lo hicieron a un ritmo sensiblemente menor. Es aquí donde el dilema entre la eficiencia ricardiana y la eficiencia schumpeteriana reaparece. Como se afirma en trabajos previos, Argentina presenta una especialización consistente con la eficiencia ricardiana en aquellos sectores o actividades que no ocupan un papel protagónico en la explicación del aumento en la productividad en los países desarrollados (Abeles et al., 2013). En contraste, se amplía la brecha tecnológica en los sectores que presentan mayores oportunidades de aumento de productividad (eficiencia schumpetariana), los cuales son la fuente de progreso técnico de los primeros.

Con lo anterior, el dilema señalado afecta la elección de una estrategia de desarrollo que permita sostener el crecimiento al tiempo que asegure la superación de la restricción externa. En un contexto de alta demanda de materias primas agrícolas, sería posible mantener un ritmo elevado de crecimiento de profundizarse la especialización ricardiana en sectores en los que las brechas tecnológicas internacionales son menores en términos relativos; ello reforzaría la heterogeneidad estructural, tal como lo dicta el principio de las ventajas comparativas estáticas. En términos dinámicos, y como se discutió en la sección 2, este perfil de especialización plantearía problemas tanto desde el punto de vista de las oportunidades de incrementos de productividad como desde el de la (menor) elasticidad-ingreso de las exportaciones.

Desde una perspectiva de eficiencia schumpeteriana, las oportunidades de aumento de productividad son menores por dos razones: de un lado, las actividades con ventajas comparativas se encuentran, por definición, más cerca de la "frontera" tecnológica que el resto; y por otro, no experimentan un dinamismo significativo dado su carácter maduro en el centro. En consecuencia, como resultado de esta tensión entre las eficiencias ricardiana y schumpeteriana, la productividad media de la industria argentina no lograría converger hacia los niveles internacionales. El crecimiento quedaría entonces limitado por la generación de divisas de un conjunto muy acotado de sectores con bajo potencial de expansión, reforzándose con ello la historia conocida de la RE, ahora desde una perspectiva dinámica. En términos de la demanda, existe evidencia según la cual los sectores intensivos en RR. NN. no 
Brechas tecnológicas y restricción externa en argentina durante los tempranos 2000: un analisis heurístico

presentan una elevada elasticidad respecto de la evolución del ingreso mundial operando la ley de Thirlwall como condicionante del crecimiento-(Abeles et al. 2013).

\section{HETEROGENEIDAD ESTRUCTURAL Y DISPERSIÓN SALARIAL: LA INDUSTRIA MANUFACTURERA EN SU ENCRUCIJADA ${ }^{5}$}

Como se analizó en ciertos trabajos previos, la persistencia de la heterogeneidad estructural plantea importantes desafíos para aquellas experiencias que pretenden periodos de crecimiento sostenidos con reducción de la desigualdad (menor dis persión salarial) ${ }^{6}$. Durante la década de 1990, la ampliación de la dispersión de las productividades entre las ramas manufactureras había coincidido con una mayor dispersión salarial entre ellas. En la primera década de los años 2000, la discrepancia entre estas dos trayectorias — de la dispersión de las brechas de productividad y de la dispersión salarial — tendió a reducir la competitividad de los sectores transables que no lograron reducir sus brechas de productividad externa, hecho que afectaría en la práctica a un amplio espectro de actividades fabriles ${ }^{7}{ }^{8}$. La mayor incidencia de los subsidios al uso industrial y comercial de energía durante la década del 2000, así como la proliferación de medidas de protección paraarancelaria, en particular a partir de 2012 (el periodo de menor dinamismo manufacturero), pueden entenderse como respuesta a esta tensión irresuelta entre una institucionalidad laboral que tendía a la homogeneidad y una estructura productiva que profundizaba su heterogeneidad'.

El gráfico 5 muestra la evolución de las dispersiones salariales y de las brechas de productividad externa entre las distintas ramas fabriles, y aporta una mirada de mediano plazo sobre esta tensión estructural ${ }^{10}$. Como lo señalan Abeles y Amar

5 Esta sección se basa en Abeles y Amar (2017, en Abeles, Cimoli y Lavarello, 2017).

6 Véase, por ejemplo, Porta et al. (2014).

$7 \quad$ La diferencia de tendencias entre un periodo y otro indica que no existe una relación biunívoca entre las diferencias de productividad sectoriales y la dispersión salarial. En línea con Altimir (1973), esa relación depende, ante todo, de una serie de factores típicamente institucionales, que no necesariamente pertenecen a la esfera productiva ni reflejan su evolución.

8 El mejor desempeño relativo en materia social que productiva fue característico de toda la región en el periodo de buenos términos del intercambio (Cepal, 2012). Una de las particularidades del caso argentino, que en América del Sur y en el mismo periodo solo se verificó en Brasil, fue el aumento de la participación asalariada en el ingreso (Abeles, Amarante y Vega, 2013).

9 En los círculos de hacedores de política, en los cuales prima una perspectiva macroeconómica o agregada, suelen plantearse estas tensiones de modo casi exclusivo en torno al nivel del tipo de cambio real. Ello tiende a relegar el problema de la heterogeneidad estructural, característico de las economías de la región, y con ello la posibilidad de un diseño más articulado entre las políticas macroeconómica e industrial y tecnológica.

10 El gráfico de cajas y bigotes permite observar la mediana, el rango intercuartil como una medida de dispersión y la amplitud entre los valores extremos de la distribución de las variables seleccionadas. 
(2017), se destacan tres hechos estilizados de la comparación entre 1997 y 2007: primero, el aumento (de la mediana) del salario real de la industria manufacturera; segundo, la disminución de la dispersión salarial; y tercero, el aumento de la dispersión de las brechas externas de productividad ${ }^{11}$. La convergencia en los distintos ingresos laborales al interior del sector manufacturero (que, en rigor, se verificó para el conjunto de los trabajadores en ese periodo) entre 1997 y 2007 constituyó uno de los principales determinantes de la mejora en la distribución del ingreso que tuvo lugar en la posconvertibilidad ${ }^{12}$.

\section{Gráfico 5. Distribución del salario real de los asalariados registrados del sector industrial y de la productividad laboral según sectores industriales*}

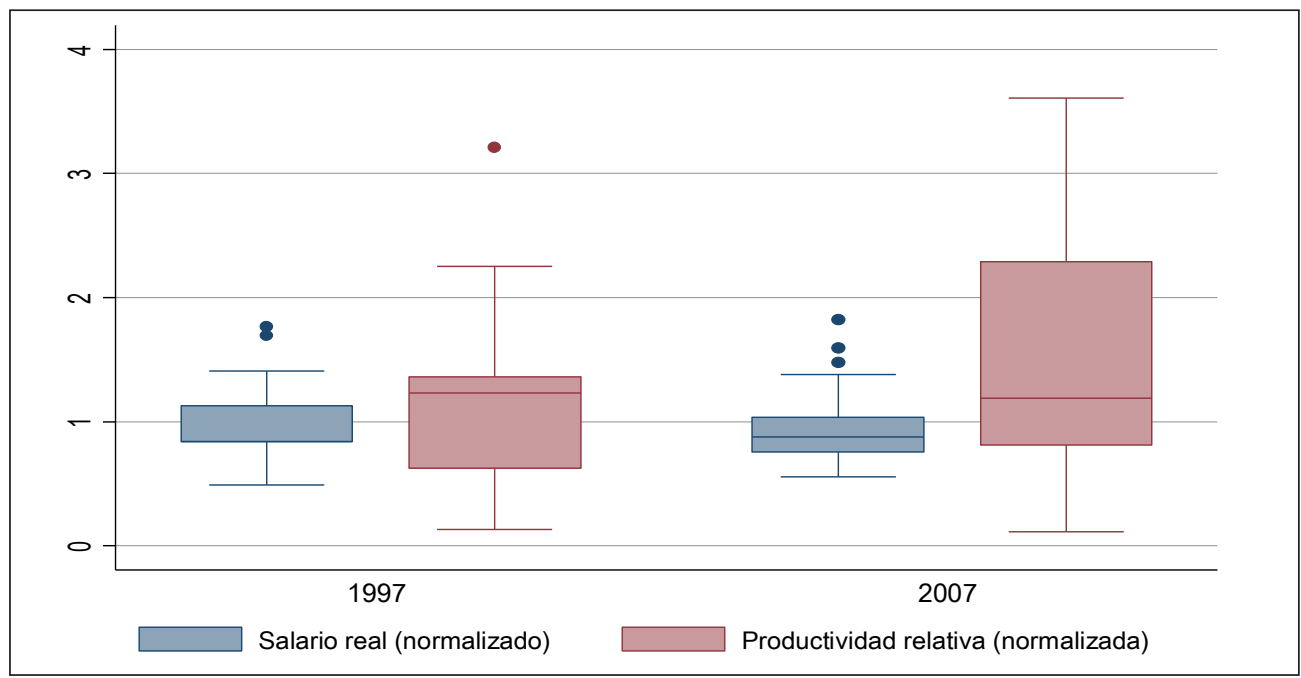

Ambos indicadores están relativizados con sus respectivos valores promedio, y ponderados por el peso de cada sector en el empleo.

Fuente: Cepal sobre la base del OEDE-MTEySS, Indec, centros de estadísticas provinciales y PADI.

En las cajas (zona sombreada del gráfico) queda representado el 50 \% de los casos que se concentran alrededor de la mediana (línea gruesa dentro de la caja), el límite inferior corresponde al valor del primer cuartil (25\%) y el límite superior al del tercero (75\%). Se denomina rango intercuartil (RI) a la distancia entre dichos valores. Los denominados "bigotes" señalan el valor que toma el sector que se encuentra a una distancia menor a 1,5 veces el RI respecto del límite de la caja. Aquellos valores que poseen un valor superior a esta medida son señalados como outliers.

11 El hecho de que la mediana (línea dentro de la caja), en el caso de los salarios reales de 1997, coincida con el límite inferior de la caja implica una importante concentración de los salarios de los sectores industriales en torno a los valores más bajos de la distribución (entre el primer y segundo cuartiles). Lo contrario ocurre con la productividad en ese año, que muestra un claro sesgo hacia los valores más altos de la distribución de esa variable.

12 La expansión de la cobertura del sistema de protección social también actuó en esa dirección, aunque con un impacto relativo menor (Trujillo y Villafañe, 2015). 
Frente a tensiones de fondo como la descrita, una posición "liberal", que suele abogar por una especialización productiva consistente con la estructura de las ventajas comparativas estáticas de la economía, tenderá a impulsar la desarticulación (o, cuanto menos, la retracción relativa) de las instituciones del mercado laboral y del sistema de protección social (que tienden a reducir las brechas de ingresos), en procura de adaptar la estructura de remuneraciones relativas a la de productividades relativas, bajo el sobreentendido de que las empresas, para sobrellevar la competencia internacional, deberían ajustarse a esas condiciones, o bien cesar sus actividades. De forma alternativa, una posición "desarrollista" si se quiere, promotora del crecimiento de determinados sectores estratégicos (por su capacidad de alejar la restricción externa al crecimiento, intensificar las interacciones sectoriales y desarrollar y difundir nuevas capacidades tecnológicas), promovería mayores esfuerzos por aumentar el ritmo de crecimiento de la productividad de los sectores schumpeterianos, en especial aquellos intensivos en ingeniería, con el fin de reducir las brechas de productividad lo más rápido posible, en aras de aproximarlas a la estructura de remuneraciones emanada de una mayor institucionalidad laboral.

\section{CONCLUSIONES}

Más allá de la evolución favorable en los términos del intercambio que ha experimentado Argentina durante la década del 2000, la RE se mantuvo como un condicionante latente al crecimiento que no tardaría en hacerse efectivo hacia el final del periodo analizado. Al igual que otras economías de América Latina, las tasas de crecimiento sostenibles desde el punto de vista del equilibrio externo siguen asociadas a factores reales que históricamente preocuparon y motivaron al pensamiento de la Cepal; en particular, el carácter especializado y heterogéneo de la estructura productiva de las economías periféricas.

Uno de los rasgos que hemos intentado subrayar corresponde al modo en que la especialización internacional según las ventajas comparativas, al reproducir el carácter especializado y heterogéneo de las estructuras productivas de los países de la región, plantea una tensión irresuelta entre las eficiencias ricardiana y schumpeteriana. Desde una perspectiva schumpeteriana, es posible sostener que el perfil de especialización basado en las ventajas comparativas estáticas plantea un problema de sustentabilidad del crecimiento en un contexto de ampliación de las brechas tecnológicas. Las políticas neoliberales de la década de 1990 (basadas en la apertura comercial, la desregulación financiera indiscriminadas y la apreciación cambiaria) profundizaron una estructura sesgada hacia actividades intensivas en RR. NN. El sector automotriz fue el único caso en el que se evidencia una reducción en la brecha de productividad más allá de los sectores intensivos en RR. NN., como 
efecto de regímenes comerciales regionales y la reestructuración de las estrategias empresariales, mas no de la apertura comercial. Desde 2003, con el colapso de la segunda experiencia neoliberal, el marco de incentivos macroeconómico procuró preservar la rentabilidad de la industria manufacturera, fundamentalmente a través de una política de tipo de cambio competitivo e impuestos diferenciales a las exportaciones de materias primas agrícolas; asimismo, favoreció un cambio incipiente en la estructura industrial hacia sectores trabajo-intensivos de baja productividad y, en menor medida, sectores intensivos en ingeniería. No obstante, estos cambios menores no se vieron acompañados por aprendizajes tecnológicos acelerados en los sectores intensivos en ingeniería, que ampliaron en forma significativa la brecha de productividad en relación con el centro. Este hecho muestra la dificultad que enfrentó el gobierno para apoyar la manufactura desde la política de CyT: predominaron en este sentido políticas horizontales que delegaban en el sector privado la adopción de paquetes tecnológicos importados en sectores intensivos en RR. NN.

Con lo dicho, la experiencia de la década del 2000 revela una vez más lo que podemos denominar "la encrucijada" de la industria manufacturera argentina: esto es, la imposibilidad histórica de sus clases dominantes de resolver la tensión entre una especialización según las ventajas comparativas o una schumpeteriana. Frente a la opción liberal, que ha fracasado en sus intentos de imponer una institucionalidad (desregulación) laboral y una estructura de remuneraciones (menos igualitaria) acorde con un modelo productivo basado esencialmente en una especialización comercial ligada a las ventajas comparativas estáticas, la opción desarrollista declamada por una multitud desorganizada de sectores subalternos, incluido parte del empresariado nacional, tampoco ha podido implementar un conjunto de políticas que, de manera consistente, permitieran cerrar las brechas de productividad que tienden a dejar inconclusa toda experiencia de crecimiento basada en la expansión de derechos. La persistencia de esta encrucijada, que se manifiesta en las crisis de divisas y de endeudamiento, abona el terreno para la reinstauración de las opciones neoliberales.

\section{BIBLIOGRAFÍA}

Abeles, Martín y Amar, Anahí (2017). La industria manufacturera argentina y su encrucijada, p. 111 -156. En: Abeles, Martín; Cimoli, Mario y Lavarello, Pablo (Editores). Manufactura y cambio estructural: Aportes para pensar la política industrial en argentina. Santiago de Chile: Cepal, 336p.

Abeles, Martín; Cimoli, Mario y Lavarello, Pablo (Eds.) (2017). Manufactura y cambio estructural: Aportes para pensar la política industrial en argentina. Santiago de Chile: Cepal, 336p. 
Brechas tecnológicas y restricción externa en argentina durante los tempranos 2000: un analisis heurístico

Abeles, Martín; Lavarello, Pablo y Montagu, Haroldo (2013). Heterogeneidad estructural y restricción externa en la economía argentina, p. 23-96. En: Infante, Ricardo y Gerstenfeld, Pascual (Editores), Hacia un desarrollo inclusivo. El caso de la argentina. Santiago de Chile: Cepal/OIT, 270p.

Cepal -Comisión Económica Para América Latina- (2007). Progreso técnico y cambio estructural en américa latina. Santiago de Chile: Cepal/IDRC,142p.

Cepal -Comisión Económica Para América Latina- (2011). Panorama de la inserción internacional de américa latina y el caribe. Santiago de Chile: Organización de las Naciones Unidas, 142p.

Chudnovsky, Daniel (1985). La difusión de tecnologías de punta en la Argentina: el caso de las máquinas herramientas con control numérico, el CAD/CAM y los robots. En: Desarrollo Económico, Vol. 24, n. 96, p. 483-515. Doi: https://doi.org/10.2307/3466919.

Chudnovsky, Daniel y Lopez, Andres (2007). Inversión extranjera directa y desarrollo: La experiencia del Mercosur. En: Revista de la Cepal, Vol. 92, 2007, p. 7-23.

Cimoli, Mario (1988). Technological Gaps and Institutional Asymmetries in a North $\square$ South Model with a Continuum of Goods. En: Metroeconomica, Vol. 39, n.ํ 3, p. 245-274.

Cimoli, Mario; Porcile, Gabriel y Rovira, Sebastián (2009). Structural Change and the BOP-Constraint: why did Latin America Fail to Converge? En: Cambridge Journal of Economics, Vol. 34, n. 2 , p. 389-411.

Cimoli, Mario y Porcile, Gabriel (2011). Technology, Heterogeneity and growth: A structuralist toolbox. MPRA Paper No. 33801. University Library of Munich, Germany.

Dornbusch, Rudiger; Fischer, Stanley y Samuelson, Paul (1977). Comparative Advantage, Trade, and Payments in a Ricardian Model with a Continuum of Goods. En: American Economic Review, Vol. 67, n. ${ }^{\circ}$, p. 823-839.

Dosi, Giovanni; D’Andrea Tyson, Laura y Zysman, John (1989). Trade, Technologies, and Development. A Framework for Discussing Japan. En: Chalmers Johnson; D'Andrea Tyson, Laura y Zysman, John (Eds.). Politics and productivity: The real story of why japan works. Cambridge, Massachusetts: Ballinger, 332p.

Dosi, Giovanni y Soete, Luc (1988). Technical change and international trade, p 401 -431. En: Dosi, Giovanni; Freeman, Christopher; Nelson, Richard; Silverberg, Gerarld y Soete, Luc (Eds). Technical change and economic theory, Laboratory of Economics and Management. Londres y Nueva York: Printer Publishers, 636p.

Katz, Jorge (1967). Características estructurales del crecimiento industrial argentino 1946-1961. En: Desarrollo Económico, Vol. 7, n. ${ }^{\circ}$ 26, p. 59-76. Doi: https://doi.org/10.2307/3465761

Katz, Jorge (1978). Creación de tecnología en el sector manufacturero argentino. En: El Trimestre Económico, Vol. 45, n.1ㅜ 177, p. 167-190.

Katz, Jorge. (1984). Domestic technological innovations and dynamic comparative advantage: Further reflections on a comparative case-study program. En: Journal of Development Economics, Vol. 16, n. ${ }^{\circ}$ 1, p. 13-37. Doi: https://doi.org/10.1016/0304-3878(84)90100-7 
Katz, Jorge y Stumpo, Giovanni (2001). Regímenes sectoriales, productividad y competitividad internacional. En: Revista de la Cepal, Vol. 75, p. 137-159.

Lavarello, Pablo (2004). Estrategias empresariales y tecnológicas de las firmas multinacionales de las industrias agroalimentarias argentinas durante los años noventa. En: Desarrollo Económico, Vol. 44, n. ${ }^{\circ}$ 174, p. 231-260.

Lavarello, Pablo y Sarabia, Marianella (2017). La política industrial en la argentina durante la década de 2000, pp. 157-199. En: Abeles, Martín; Cimoli, Mario y Lavarello, Pablo (Editores). Manufactura y cambio estructural: Aportes para pensar la política industrial en argentina. Santiago de Chile: Cepal, 336p.

Patel, Parimal y Pavitt, Keith (1994). The Continuing, Widespread (and neglected) Importance of Improvements in Mechanical Technologies. En: Research Policy, Vol. 23, n. ${ }^{\circ}$ 5, p. 533-545. Doi: https://doi.org/10.1016/0048-7333(94)01004-8"

Pinto, Anibal (1970). Naturaleza e implicancias de la 'heterogeneidad estructural' en América Latina. En: El Trimestre Económico, Vol. 37, n.¹45, p. 83-100.

Porta, Fernando; Santarcángelo, Juan y Schteingart, Daniel (2014). Excedente y desarrollo industrial en Argentina: Situación y desafíos, Cefidar, DT 59.

Prebisch, Raúl (1949). El desarrollo económico de la América Latina y algunos de sus principales problemas. En: Estudio económico de la América Latina. Naciones Unidas.

Prebisch, Raúl (1962). The Economic Development of Latin America and its Principal Problems. En: Economic Bulletin for Latin America, Vol. 7, n. 1, pp. 1-22.

Rosenberg, Nathan (1974). Science, Invention and Economic Growth. En: Economic Journal, Vol. 84, n. ${ }^{\circ} 333$, p. $90-108$.

Sunkel, Osvaldo (1970). Desarrollo, subdesarrollo, dependencia, marginación y desigualdades espaciales: hacia un enfoque totalizante. En: Revista de Estudios Urbanos Regionales, Vol. 1, n. $^{\circ} 1$, p. 13-49.

Thirlwall, Anthony (1979). The balance of payments constraint as an explanation of international growth rate differences. En: BNL Quarterly Review, Vol. 32. n.128, p. 45-53. 